\title{
Computational Analysis of Goal-Oriented Adaptive Strategies based on Several Error Representations
}

\author{
Serge Prudhomme*, Diane Guignard ${ }^{\dagger}$, and Vincent Darrigrand ${ }^{\ddagger}$ \\ *Department of Mathematics and Industrial Engineering \\ Polytechnique Montréal \\ C.P. 6079, succ. Centre-ville, Montréal, QC, Canada H3C 3A7 \\ e-mail: serge.prudhomme@polymtl.ca \\ web page: https://www.polymtl.ca/expertises/en/prudhomme-serge \\ ${ }^{\dagger}$ Department of Mathematics and Statistics \\ University of Ottawa \\ 150 Louis-Pasteur Pvt, Ottawa, ON, Canada K1N 6N5 \\ e-mail: dguignar@uottawa.ca - web page: https://mysite.science.uottawa.ca/dguignar/ \\ $\ddagger$ Institut de Recherche en Informatique (IRIT) \\ 2, rue Camichel, BP 7122, 31071 Toulouse Cedex 7, France \\ e-mail: vincent.darrigrand@irit.fr
}

\begin{abstract}
Goal-oriented error estimation and adaptivity provides a collection of techniques to control approximations of a specific quantity of interest (QoI), defined as a functional of the solution to some initial or boundary-value problem, the so-called primal problem. Using the adjoint problem, the error in the QoI can be represented in several ways in terms of the error in either the primal or the adjoint solutions, or both. In practice, computable a posteriori goal-oriented error estimates are obtained using solutions computed, for instance, on a finer mesh. For adaptive purposes, the error estimates should be decomposed into local contributions. However, the decomposition is not unique and the local refinement indicators may vary from one decomposition to another. This observation immediately implies that goal-oriented adaptive algorithms may perform differently depending upon the selected representation and decomposition. The goal of the presentation will be to compare the performance of adaptive procedures when using some of the proposed error decompositions.
\end{abstract}

